\title{
Editorial: Stress metabolism of plants
}

\author{
Bhumi Nath Tripathi
}

Received: 6 August 2010 / Accepted: 6 August 2010

(C) Springer-Verlag 2010

The present issue of Protoplasma covers the stress metabolism of plants. The stresses can be divided in two broad categories: abiotic stress, induced by various complex environmental conditions, such as, strong light, high and low temperatures, radiation, mineral deficiency/toxicity, heavy metals, metalloids, salinity, drought, flooding, hypoxia, etc. and biotic stress including pathogen attack, herbivory, etc. These stresses are known to adversely affect the plant metabolism and thereby greatly diminish the crop yield. Pervasiveness of these stresses throughout the world and their severity on crop yield has spurred a great deal of research around the globe. Therefore, understanding the stress metabolism of plants is now considered to be one of the most important topics of the plant biology.

The above-mentioned stresses impose their toxicity to plants through a variety of well-studied stress-specific mechanisms. The toxicity of all these stresses eventually leads to the development of oxidative stress (cellular damage caused by reactive oxygen species) in plants. A trickle of reactive oxygen species is constantly produced in all aerobic life forms even during the course of normal metabolism. But adverse environmental conditions directly or indirectly accelerate the generation of reactive oxygen species in plant cell. In order to avoid the harmful effects of reactive oxygen species, plant cell maintains a dynamic equilibrium between the formation and destruction of reactive oxygen species, called as redox-homeostasis. Characterization of redoxhomeostasis and oxidative metabolism is an important research area of stress plant biology. Substantial information has been accumulated on various aspects of redox-homeostasis

B. N. Tripathi $(\bowtie)$

Department of Bioscience and Biotechnology,

Banasthali University,

Banasthali 304022, Rajasthan, India

e-mail: bhuminathtripathi@hotmail.com in plants during stress conditions. Stresses trigger the accumulation of a variety of molecules in the plant cell. Among them, induction of compatible solutes, e.g., proline, glycine betaine, mannitol, etc., have received considerable attention due to their widespread occurrence during multiple stresses. Compatible solutes, especially proline, are known to confer tolerance to plants against a variety of stresses by protecting membrane damage and protein, maintaining osmotic balance, and scavenging free radicals, etc.

Our knowledge about the stress responses of plants has improved significantly during the last decade, namely the postgenome-era. Availability of the whole genome sequences of several plants, e.g., Arabidopsis, rice, etc., enabled genomewide gene expression profiling in response to various stresses. Using microarray technology, stress-responsive genes have now been identified more comprehensively than even before. Transcriptomic studies revealed the relationships among stress-regulated transcripts and also enabled the prediction of their cis-regulatory elements. The products of the stressinducible genes identified in transcriptomic studies can be classified into two groups, i.e., elements directly involved in stress tolerance and regulatory elements for intracellular stress signaling and stress-inducible gene expression. The discovery of functional small RNAs, such as micro-RNA and small interference RNA, has further elucidated the complex regulatory network of plants exposed to stresses. Despite the existence of promising knowledge and techniques for the improvement of stress tolerance, further research are required to completely understand the whole stress response system of plants. Integration of genomics, proteomics, and metabolomics data would greatly advance our understanding of the overall stress responses in plants.

Various aspects of the stress metabolism of plants ranging from physiological and biochemical to molecular analyses have been complied in the present special issue of the Protoplasma. 